\title{
Large-Eddy Simulation of Heat Transfer from a Single Cube Mounted on a Very Rough Wall
}

3 V.B.L. Boppana · Z-T Xie · I.P. Castro

\begin{abstract}
The local thermal effects in the wake of a single cube with a strong heated rear face, representing a large building in an urban area, are studied using large-eddy simulations (LES) for various degrees of heating, which are characterized by the local Richardson number, Ri. New wall models are implemented for momentum and temperature and comparison of the flow and thermal fields with the wind-tunnel data of Richards et al. (Journal of Wind Engineering and Industrial Aerodynamics, 2006, 94, 621-636) shows fair agreement. Buoyancy effects are quite evident at low Ri and a significant increase in the turbulence levels is observed for such flows. Apart from the comparisons with experiments, further analysis included the estimation of the thermal boundary-layer thickness and heat transfer coefficient for all Ri. For sufficiently strong heating, the heat transfer coefficient at the leeward face is found to be higher than the roof surface. This suggests that, beyond a certain $R i$ value, buoyancy forces from the former surface dominate the strong streamwise convection of the latter. Quadrant analysis along the shear layer behind the cube showed that the strength of sweeps that contribute to momentum flux is considerably enhanced by heating. The contribution of different quadrants to the heat flux is found to be very different to that of the momentum flux for lower $R i$.
\end{abstract}

3 Keywords Heat flux $\cdot$ Rough wall $\cdot$ Single cube $\cdot$ Wall model

\section{Introduction}

25 Rapid increase in urbanization over the last few decades has given rise to several 26 challenging problems in the field of Urban meteorology. One of the challenges is un27 derstanding and modelling the thermal effects in urban flows. These can arise because

V. B. L. Boppana · Zheng-Tong Xie · Ian P. Castro

School of Engineering Sciences

University of Southampton

Tel: $+44(0) 2380592320$

Fax: +44(0)23 80593058

E-mail: V.B.Boppana@soton.ac.uk 
the background atmosphere is non-neutral or because of localized surface heating of buildings in the urban canopy. This paper focusses on the latter.

There have been various investigations of thermal effects using wind-tunnel and/or field experiments or numerical computations. Several of the latter have used Reynolds Averaged Navier-Stokes (RANS) and large-eddy simulations (LES) of two-dimensional (2-D) canyons in neutral atmospheres. These have shown that local differential heating within the urban canopy has various effects on the canyon flow and pollutant dispersion (Sini et al., 1996; Kim and Baik, 1999; Li et al., 2010; Cheng and Liu, 2011; Cai, 2012; Park et al., 2012). In these studies, it was observed that buoyancy forces due to ground or leeward wall heating strengthen the intensity of the circulation within the canyon, resulting in increases in the vertical exchange rates of passive pollutants. In a three-dimensional (3-D) RANS study by Dimitrova et al. (2009) on a geometry that resembles an urban area in Lisbon, Portugal, it was observed that the airflow within the canyon was significantly altered by the solar heating of the building surfaces. These computations clearly indicated that surface heating has significant impact on the flow inside the canyons. However, some field studies (Louka et al., 2002; Offerle et al., 2007; Idczak et al., 2007) suggest that the buoyancy effects on the canyon flow are not as significant as those suggested by the computations. This could be attributed either to inadequate numerical representation of flow or thermal physics near the wall or too simplified a model geometry, although it should be noted that point measurements in the field are inevitably very limited. A few field studies also suggest, firstly, that large horizontal thermal gradients exist in the vicinity of walls heated by solar radiation (Louka et al., 2002) and, secondly, that the heat transfer is very intermittent within the wall boundary layer of a leeward-heated wall (Nottrott et al., 2011). This implies that grid resolution in the computations has to be very fine, or that sophisticated near-wall models need to be developed.

Therefore, with the broader aim of simulating canopy stability effects in urban areas and obtaining accurate estimates of the heat flux from building walls by resolving the thermal boundary layer, we first simulated dispersion from a passive scalar area source in an array of roughness elements using LES (Boppana et al., 2010). This is analogous to dispersion from surface heat sources when the resulting temperature differences are sufficiently small that they do not influence the flow dynamics. These simulations showed good agreement with the wind-tunnel experiments of Pascheke et al. (2008). Our next step was then to use LES to simulate the buoyancy effects in urban flows - i.e. when temperature differences do influence the flow dynamics - and this paper presents the results. A wind-tunnel study by Richards et al. (2006), in which the leeward face of a cube was heated, was considered to be appropriate for validation of our numerical methodology. Hereafter, 'experiments' refer to this wind-tunnel study unless specified otherwise.

The numerical details of the computational study are outlined in Sect. 2. In Sect. 3, results of computations from the heated cube are compared with the experiments and some details on the thermal boundary-layer thickness, heat transfer coefficient and quadrant analysis of momentum and heat fluxes are presented. Finally, conclusions are given in Sect. 4. 


\section{Numerical Details and Settings}

\subsection{Governing equations and boundary conditions}

The filtered continuity and Navier-Stokes equations governing unsteady incompressible flow are

$$
\begin{aligned}
\frac{\partial u_{i}}{\partial x_{i}} & =0 \\
\text { and } \frac{\partial u_{i}}{\partial t}+\frac{\partial u_{i} u_{j}}{\partial x_{j}} & =-\frac{\partial}{\partial x_{i}}\left(\frac{p}{\rho}\right)+\frac{\partial}{\partial x_{j}}\left(\frac{\tau_{i j}}{\rho}+v \frac{\partial u_{i}}{\partial x_{j}}\right)+f \delta_{i 3} .
\end{aligned}
$$

The resolved-scale velocity and pressure are respectively given by $u_{i}$ and $p$ with $u$, $v$ and $w$ the streamwise, lateral and vertical velocity components respectively. $f \delta_{i 3}$ is the body force due to thermal buoyancy and is estimated using the Boussinesq approximation; $\rho$ and $v$ are the density and kinematic viscosity of the fluid; $\tau_{i j}$ is the subgrid-scale (SGS) Reynolds stress and was handled using the Smagorinsky model in conjunction with a Lilly damping function near the walls. We set Smagorinsky's constant $C_{S}=0.1$.

At the inlet, the inflow method proposed by Xie and Castro (2008) was used as the boundary condition. In the lateral $(y)$ direction, a periodic boundary condition was employed and at the outlet, a zero normal gradient for $u, v$ and $w$ with a mass balance correction was imposed. Stress free conditions were imposed at the top of the domain. The boundary condition for the bottom surface is treated with a rough-wall model, with details given in Sect. 2.3.

The filtered governing equation for temperature is

$$
\frac{\partial \theta}{\partial t}+\frac{\partial u_{j} \theta}{\partial x_{j}}=\frac{\partial}{\partial x_{j}}\left(\left(k_{s}+k_{m}\right) \frac{\partial \theta}{\partial x_{j}}\right),
$$

where $\theta$ is the resolved-scale temperature. $k_{S}$ is the subgrid turbulent diffusivity and is given by $v_{s} / P r_{s}$, where $v_{s}$ is the subgrid viscosity and $P r_{s}$ is the subgrid Prandtl number whose value was set to $0.9 . k_{m}$ is the molecular diffusivity and is defined as $v / \mathrm{Pr}_{m}$. The gradient (normal to the boundary) of the temperature was set to zero at the top of the domain and at the outlet.

A finite volume approach was followed to discretize the flow and temperature equations. The monotone advection and reconstruction scheme (?) with a blending factor of 0.9 was used for the spatial convective terms and the central difference scheme is used for spatial diffusive terms of (1) and (2). A second-order backward implicit scheme was used for discretizing the time-dependent term. The initial and average durations of the simulations were approximately 120 and $200 L_{x} / u_{\text {ref }}$ respectively, where $L_{x}$ is the streamwise domain length and $u_{\text {ref }}$ is the time- and spatiallyaveraged streamwise velocity at the top of the domain. Tests showed that this was sufficient to produce statistically converged data. All the computations were carried out using ? version 4.14 . 


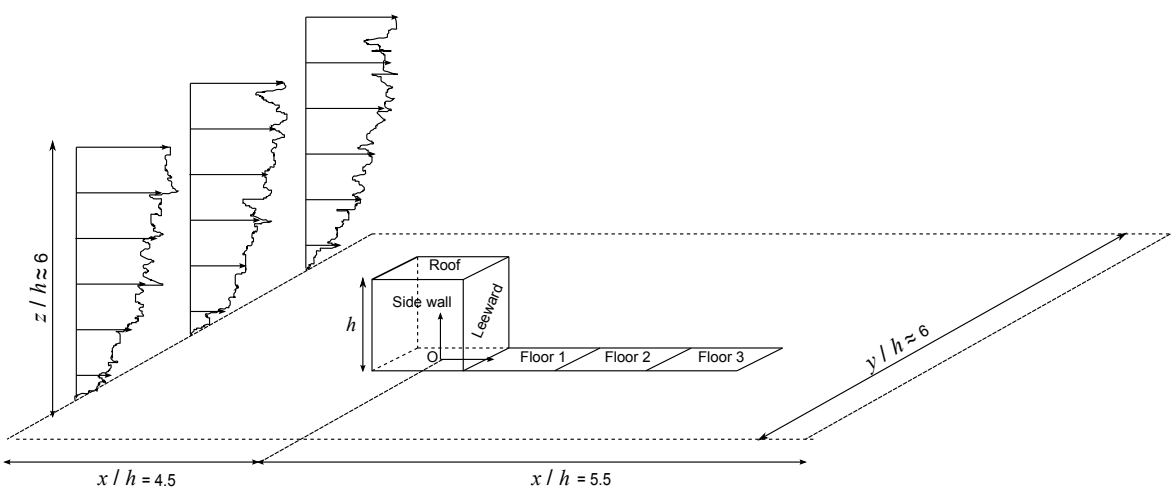

Fig. 1 Sketch of 3-D view of the computational domain with a single cube. The heated surfaces are shown.

\subsection{Settings}

In the wind-tunnel experiments, the cube height $(h)$ was $0.19 \mathrm{~m}$; the cube was made of plaster of Paris and an aluminium plate of $2 \mathrm{~mm}$ thickness was fitted on its leeward face. This plate was heated from inside using a ceramic radiant heater that was powered by an autotransformer. The leeward face thus had neither constant temperature nor constant heat flux. This imposed some difficulty in specifying the exact heat boundary condition in our computations. However, temperature was measured at two points approximately equi-distant either side of the centre point on the leeward face in the experiments. The average of these measurements is given in Table 1 of Richards et al. (2006) and this value was specified as the uniform temperature on the leeward face in the present computations. In the experiments, thermocouples were also mounted on the top surface, defined hereafter as the 'roof', and two streamwise faces (also called 'side' faces) of the cube, and on the bottom floor behind the cube. The measured values were again specified as uniform temperature boundary conditions in the computations (see later). Unlike the roof and side faces, it is not clear from Richards et al. (2006) at what location the temperature was measured on the bottom floor. We have assumed that the temperature on the floor decreases with increasing distance from the leeward face. Therefore, as an approximation of this, different surface temperatures were specified uniformly in the three regions of size $h \times h$ shown in Fig. 1; the values are tabulated in Table 1. In the experiments there were no roughness elements positioned in the surrounding region of the cube; to simulate this, the third square region denoted by 'floor 3' in Fig. 1 and a region of size $2 h \times 4 h$, positioned so that its upstream edge was a distance of about $h$ ahead of the cube's front face, had no roughness elements and so was smooth. The remaining region of the bottom surface of the domain was treated as rough. Except for the heated regions on the bottom wall, an adiabatic boundary condition was specified on the remaining surface. Possible implications of these various assumptions are discussed in due course. In all computations, the reference temperature $\theta_{\text {ref }}$ was $293 \mathrm{~K}$. The Reynolds number based on the cube height and the streamwise velocity at that height was approximately 6000 . 


\begin{tabular}{lc}
\hline Surface & $\theta_{\text {wall }}(\mathrm{K})$ \\
\hline Leeward & 445 \\
Roof & 319 \\
Side wall & 307 \\
Floor 1 & 321 \\
Floor 2 & 307 \\
Floor 3 & 300 \\
\hline
\end{tabular}

\begin{tabular}{cc}
\hline$R i$ & $g$ \\
\hline 0.00 & 9.81 \\
-0.02 & 0.38 \\
-0.24 & 3.50 \\
-0.66 & 9.81 \\
-2.00 & 29.02 \\
\hline
\end{tabular}

Table 1 Specified temperature on different surfaces.

Table 2 Variation of $g$ with $R i$.

The domain size for these computations was $10 h \times 6.2 h \times 6 h$ because this proved sufficient in previous related studies (e.g. Lim et al., 2009). Except for the uniform resolution of $h / 19$ in the $x$ and $y$ directions on the cube surface, the rest of the domain had non-uniform spacing that followed a geometric progression. In the $x$ and $y$ directions, the resolution was of size $h / 19$ near the cube and expanded to a resolution of $h / 7$ at the boundaries, such that the mesh expansion ratio did not exceed 1.04. In the vertical direction, the resolution decreased from $h / 35$ at the cube height to $h / 7$ at $z / h \approx 0$, such that the mesh expansion ratio was 1.13 ; the resolution again decreased from the cube height to the domain top $(h / 7)$ with a mesh expansion ratio of 1.02. The resolution has to be relatively coarse at the bottom wall because the rough-wall model requires the first wall grid to lie within the (notional) logarithmic layer i.e. $30<z_{1}^{+}<200$ (where $z_{1}^{+}=u_{\tau} z_{1} / v, u_{\tau}$ is the wall friction velocity and $z_{1}$ is the distance from the wall to the centre of the first grid).

In order to understand the local thermal effects on the flow, experiments were conducted for various $R i$, defined as

$$
R i=\frac{\beta g h\left(\theta_{\mathrm{ref}}-\theta_{\mathrm{wall}}\right)}{u_{\mathrm{ref}}^{2}} .
$$

Here $\beta$ is the coefficient of thermal expansion, $g$ is the acceleration due to gravity and $\theta_{\text {wall }}$ is the mean wall temperature on the leeward face. ${ }^{1}$ In order to maintain consistency in comparisons for different cases of $R i, \theta_{\text {wall }}$ and $\theta_{\text {ref }}$ were fixed and $g$ was changed accordingly; the resulting values are shown in Table 2.

\subsection{More details on wall boundary conditions}

\subsubsection{Rough-wall momentum model}

In the experiments, sharp edged roughness elements (almost similar to vertical flat plates) comprising two different heights $(h / 2.375$ and $h / 3.8)$ were arranged in the form of an aligned array on the bottom floor of the wind tunnel. Such an arrangement crudely represents the urban roughness. In the current computations, the turbulence created by these roughness elements was represented by a rough-wall model developed by Xie et al. (2004). This model is based on the principle of injecting energy

1 Note that in the experiments, the reference velocity for $R i$ was taken as the mean velocity at $(x / h, y / h, z / h)=(-1.5,0,1.25)$, rather than our $u_{\text {ref }}$. 
into the resolved velocity field. According to this model, the local wall shear stress is given by

$$
\frac{\tau_{z x}}{u_{*}^{2}}=\frac{1}{U_{a}}\left[\langle u\rangle+(u-\langle u\rangle)\left(\beta_{0}+\beta_{1} \frac{|u-\langle u\rangle|}{u_{*}}+\beta_{2} \frac{|u-\langle u\rangle|^{2}}{u_{*}^{2}}+\ldots+\beta_{l} \frac{|u-\langle u\rangle|^{l}}{u_{*}^{l}}\right)\right],
$$

where \langle\rangle represents averaging in the horizontal plane, $u$ is the streamwise velocity at the first grid point from the wall, $U_{a}$ is the mean streamwise velocity at the same location, $u_{*}$ is the friction velocity and $\beta_{k}$ (where $k=0,1 \ldots l$ ) is the damping factor that ranges between 0 and 1 . The value of $\beta_{k}$ can be obtained from the similarity laws or measurements. A similar relation can be written for instantaneous shear stress in the spanwise direction $\left(\tau_{z y}\right)$. Unlike Xie et al. (2004), who estimated the value of $\beta_{2}$ from wind-tunnel near-wall data, such data are not known from the experiments of Richards et al. (2006) and therefore the choice of $\beta_{k}$ remains an issue. However, we tested various values of $\beta_{1}$ by assuming that the contribution from other $\beta$ terms are small. $\beta_{1}=0.01$ seemed to be reasonable (with $\beta_{0}=0$ ), as values larger than this yielded steep gradients in the turbulent kinetic energy near the wall and smaller values did not yield sufficient turbulence to represent adequately the 'very' rough wall.

\subsubsection{Smooth-wall momentum model}

The logarithmic law is commonly employed to incorporate the near-wall shear stress if the near-wall grid lies in the logarithmic region. But such an approximation does not yield accurate skin-friction coefficients, especially in complex flow regions. In an attempt to address such issues, Cabot and Moin (1999) developed a wall model that includes solving boundary-layer equations on an embedded finer mesh between the wall and the near-wall grid, thereby improving the near-wall modelling. Such a method was implemented successfully on various complex problems such as boundarylayer flows past an aerofoil trailing edge (Wang and Moin, 2002) and backward facing step flow (Rani et al., 2009). A brief description of this method is given here; for more details, refer to Wang and Moin (2002). The wall shear stress is obtained by solving the following turbulent boundary-layer equations for the two tangential velocity components on an embedded mesh between the wall and the first grid point.

$$
\frac{\partial}{\partial x_{n}}\left(v+v_{t}\right) \frac{\partial u_{i}}{\partial x_{n}}=F_{i}
$$

$$
\text { lwhere } F_{i}=\frac{1}{\rho} \frac{\partial p}{\partial x_{i}}-f \delta_{i 3}+\frac{\partial u_{i}}{\partial t}+\frac{\partial}{\partial x_{j}} u_{i} u_{j}
$$

for given $n \in \mathbf{J}, \forall i \in \mathbf{J} \backslash\{n\}, \forall j \in \mathbf{J}$ where $\mathbf{J}=\{1,2,3\}$.

Here $n$ denotes the wall-normal direction. Wang and Moin (2002) computed the eddy viscosity $v_{t}$ using the mixing-length model with near-wall damping, so that

$$
\frac{v_{t}}{v}=\kappa z_{w}^{+}\left(1-e^{-z_{w}^{+} / A}\right)^{2}
$$


where $z_{w}^{+}$is the distance to the wall in wall units, $A=19$ and $\kappa$ is the model coefficient. If the transient and convective terms are ignored in Eq. 6, Eq. 5 reduces to an ordinary differential equation, which upon integration from the wall to the first grid point yields the following.

$$
\tau_{w i}=\left.\mu \frac{\partial u_{i}}{\partial x_{n}}\right|_{x_{n}=0}=\frac{\rho}{\int_{0}^{\eta} \frac{\mathrm{d} x_{n}}{v+v_{t}}}\left\{u_{\eta_{i}}-F_{i} \int_{0}^{\eta} \frac{x_{n}}{v+v_{t}} \mathrm{~d} x_{n}\right\}
$$

where $\eta$ is the first wall-normal grid point.

In the current computations, the wall shear-stress components were calculated using Eq. 8. $v_{t}$ was obtained from the mixing-length model given by Eq. 7 in which $\kappa$ was set to a constant value of 0.4. Similar to Wang and Moin (2002), the local friction velocity $u_{\tau}$ at each timestep was obtained from the previous timestep using the term $\left[\left(\tau_{w 1} / \rho\right)^{2}+\left(\tau_{w 2} / \rho\right)^{2}\right]^{1 / 4}$.

\subsubsection{Smooth-wall temperature model}

Similar to the momentum wall model above, a wall model for the temperature can be derived from the temperature transport equation given by Eq. 2. Ignoring the transient and convection terms and integrating the diffusion term twice gives the wall heat flux $q_{w}$ as

$$
q_{w}=\left.\frac{\mu}{P r_{m}} \frac{\partial \theta}{\partial x_{n}}\right|_{x_{n}=0}=\frac{\rho c_{p}}{\int_{0}^{\eta} \frac{\mathrm{d} x_{n}}{k_{t}+k_{m}}}\left(\theta_{\eta}-\theta_{\mathrm{wall}}\right),
$$

where $c_{p}$ is the specific heat capacity, $\theta_{\eta}$ and $\theta_{\text {wall }}$ are the temperatures at the nearwall grid point and the wall, respectively. Such a wall model was also derived and implemented by Rani et al. (2009). In the current computations, $\operatorname{Pr}_{t}\left(=\mu_{t} / k_{t}\right)$ was set to a constant value of 0.9 and the heat transfer coefficient specified at the wall was given by Eq. 9 .

In order to assess the performance of the above mentioned temperature and momentum (Sect. 2.3.2) wall models for the smooth wall, they were initially implemented on flow past four staggered cubes with a passive scalar on the bottom wall surface. The details on geometry and boundary conditions are given in Boppana et al. (2010). The resulting time and spatial average scalar and streamwise velocity profiles were then compared with fine resolution LES (this is close to direct numerical simulations at the wall) and coarse resolution $(h / 16)$ with the standard logarithmic law at the wall. With the above smooth wall models, although $6 \%$ improvement is observed at the bottom wall streamwise velocity, approximately $34 \%$ improvement is observed in the near-wall scalar. Therefore, in the current set of large-eddy simulations, these smooth wall models are used on the cube and heated floor surfaces.

\section{Results}

As mentioned in Sect. 2.2, for the flow past a heated cube on a very rough wall, the thermal effects were investigated for $R i=0,-0.02,-0.24,-0.66$ and -2.0 . This 
range of $R i$ includes forced and mixed convection in the flow around the cube. The approach flow is maintained similar to experiments and its modelling details are given in the Appendix. The flow and thermal fields behind the cube are compared with the experiments in Sect. 3.1 and further analysis on these fields is presented in Sect. 3.2.

\subsection{Comparisons with experiments}

Results are shown with the experimental data mostly for $R i=-0.66$; where necessary, the results for other $R i$ cases will be explained. The normalized time-averaged streamwise velocity, kinetic energy and temperature profiles behind the cube and at two different spanwise locations are shown in Figs. 2, 3 and 5 respectively.

The streamwise velocities obtained from LES are found to be in fair agreement with experiments for both $R i=0$ and -0.66 . However, a notable observation is that the computed velocities above the cube height are lower than the experimental data at all locations. The measured velocity profile at $x / h=-3.5$ follows approximately a power-law behaviour and the experimental data slightly exceeds the power-law values in the region $0.6<z / h<1.6$. This could partly explain the differences seen in Fig. 2. It is observed that below the cube height, heating enhanced the magnitude of the streamwise velocity, especially beyond the recirculation region $(x / h=1.5)$.

Figure 3 shows that the turbulent kinetic energy from simulations is also in fair agreement with the experiments. Heating causes a notable increase in the kinetic energy, especially for $z / h<1$. Above the cube height, the LES shows higher kinetic energy than experiments at all downstream locations. This could be due to slightly larger TKE than the experiments at the inlet for $z / h<2$ (not shown here). Contours of the normalized TKE and mean velocity vector field behind the cube and at the symmetry plane (i.e $y / h=0$ ) are shown in the Fig. 4 for four different cases of heating. The figure clearly shows the increase in TKE with the decrease in $R i$, and this behaviour is not limited to the wake of the cube but extends towards the shear layer and above, especially for $R i=-2$. In the cube wake, increasing buoyancy clearly leads to increase in velocity vector magnitude.

The simulations for $R i=-0.66$ in Fig. 5 show lower values of normalized temperatures than the experiments at all downstream locations except at $x / h=0.55$. The difference between simulation and experiment is large only below the cube height. It cannot be entirely attributed to the small differences in the flow field between simulations and experiments, for reasons explained below. The figure also shows the temperatures that were obtained by not using the temperature and momentum wall models described in Sect. 2.3. Clearly, the temperature estimation is improved with these wall models and so they were used in all the subsequent computations.

It was mentioned in Sect. 2.2 that the heating in the experiments had neither constant heat flux nor constant temperature on the leeward face of the cube; the total input heat flux was also not measured. This imposes a challenge in making appropriate comparisons of simulated temperature field with the experiments. However, with only marginal differences seen in the velocity field, we can assume that the total heat flux must be the same far downstream of the cube in both LES and wind-tunnel experiments (and of course equal to the total input heat flux at the various heated sur- 


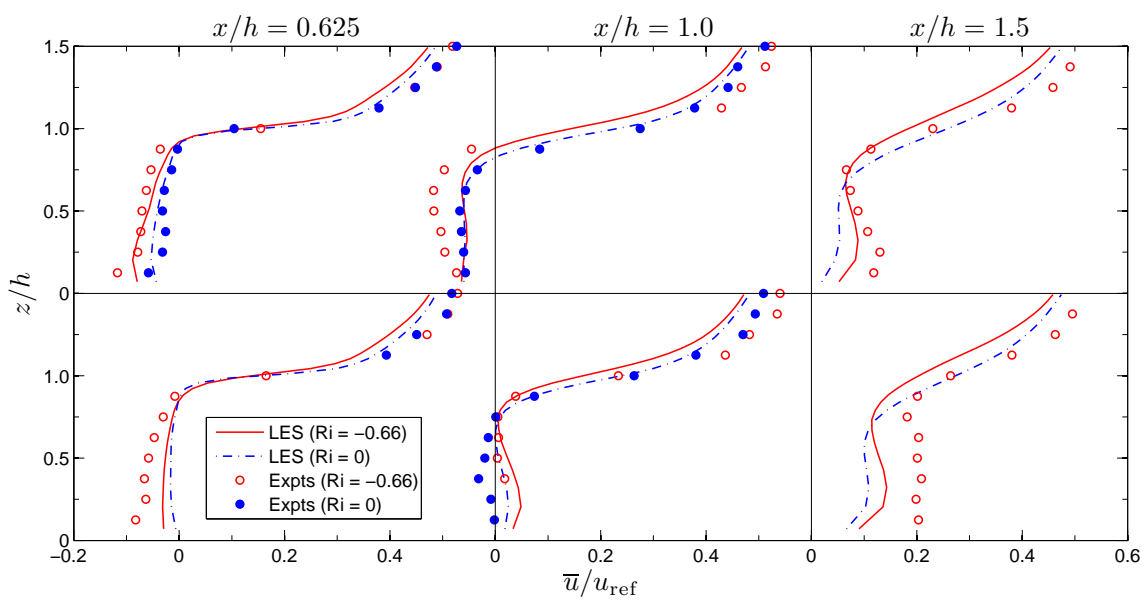

Fig. 2 Normalized mean streamwise velocity profiles at different downstream locations behind the cube for $R i=0$ and -0.66 : Top row at $y / h=0$, bottom row - average of data at $y / h= \pm 0.25$.

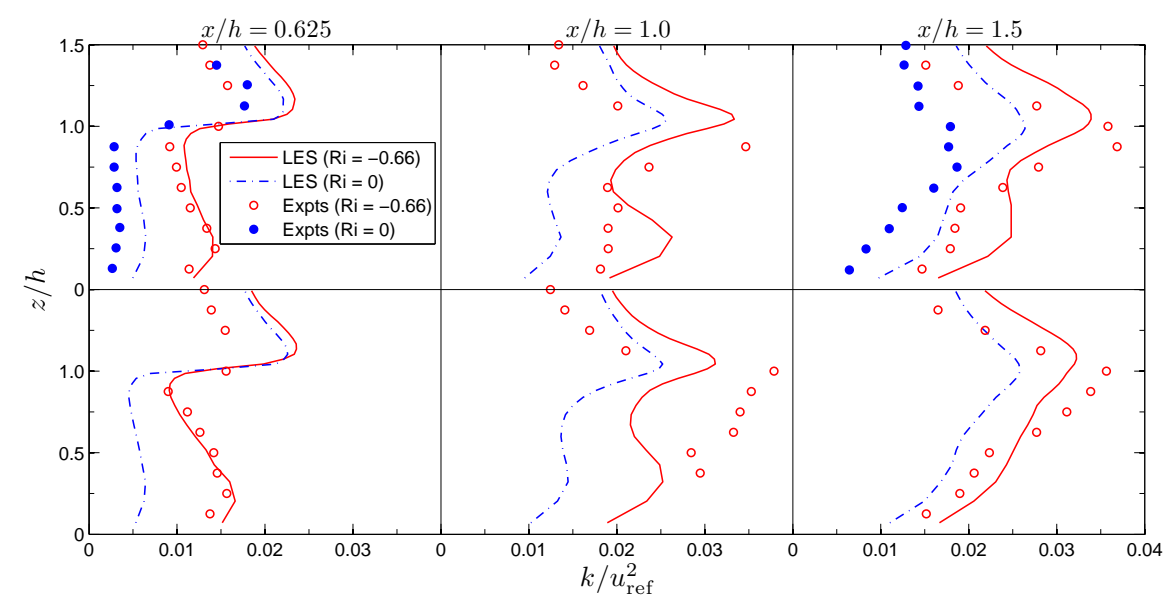

Fig. 3 Normalized mean turbulent kinetic energy profiles at different downstream locations behind the cube for $R i=0$ and -0.66 : Top row at $y / h=0$, bottom row - average of data at $y / h= \pm 0.25$.

faces). Since the data available from the experiments were insufficient to allow direct estimation of this total heat flux, we attempted to match the temperature profiles in the wake of the cube in order to estimate the total heat flux in the experiments.

Let $q$ be the total (input) heat flux obtained from LES with the constant temperature boundary conditions (detailed earlier). Normalizing the mean temperature using this heat flux yields profiles shown in Fig. 6. Since the heat flux in the experiments is unknown, several trials have shown that the total heat flux in the experiments could be $q_{\text {est }}=1.8 q$. This is because, reasonable agreement between experiments and computations can be seen in Fig. 6 when the former is normalized with $q_{\text {est }}$. Therefore, an 


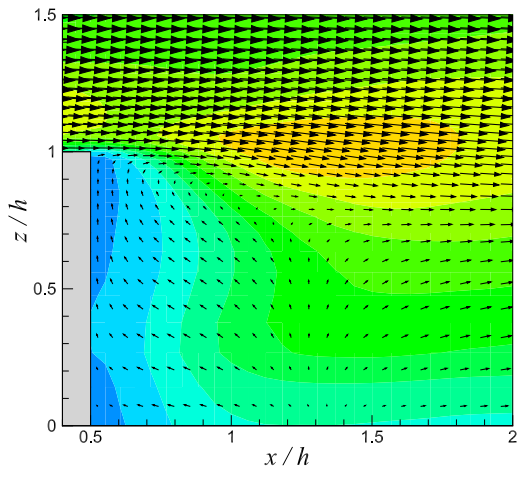

(a) $R i=0$

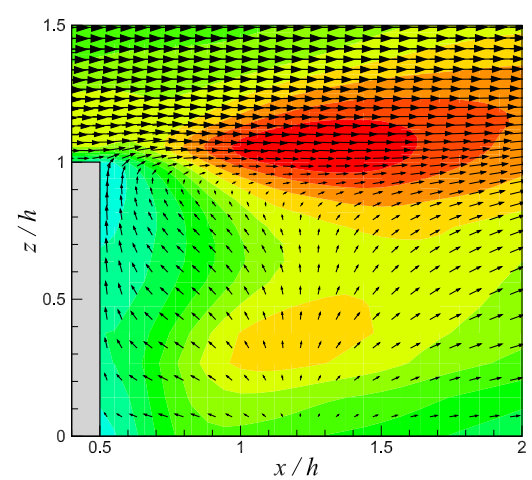

(c) $R i=-0.66$

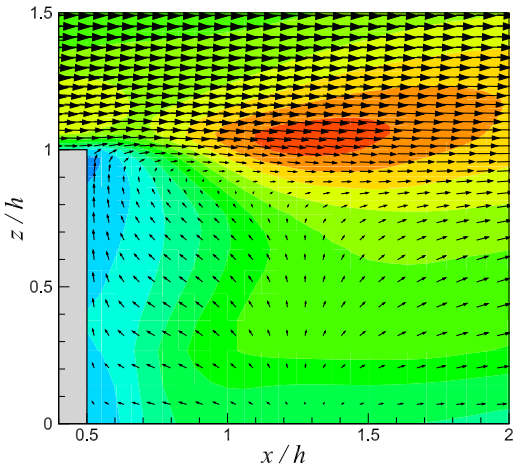

(b) $R i=-0.24$

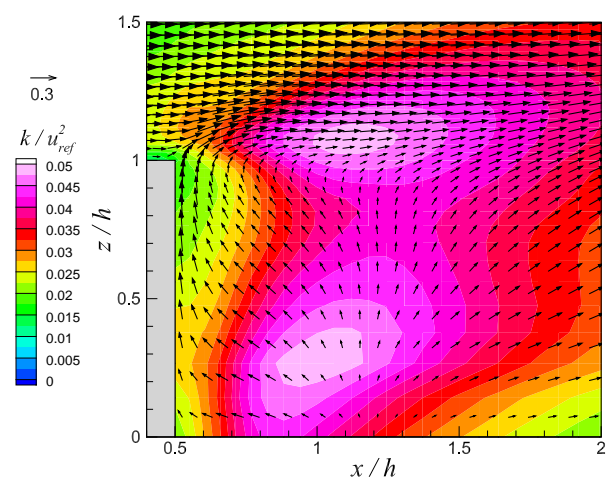

(d) $R i=-2$

Fig. 4 Contours of the normalized TKE and mean velocity vectors in the wake of the cube.

LES was performed in order to check the sensitivity of the type of heat boundary condition and $q_{\text {est }}$ on the flux normalized temperature profiles. The resulting normalized temperature profiles shown in Fig. 6 confirms the reasonableness of the estimate of $q_{\text {est }}$ for the experimental heat flux and insensitivity to the type of heat boundary condition. In order to assess the importance of heating the floor surfaces shown in Fig. 1, additional computations were done by heating only the leeward face of the cube. In the resulting normalized temperature profiles (not shown here), the near-wall temperatures were found to be severely under-predicted, thereby justifying the bottom floor heating in the current set of computations .

Figure 7 shows the contours of the flux-normalized mean temperature for different $R i$ at $z / h=0.5$. There are two striking features in this figure. Firstly, with decrease in $R i$ the normalized temperatures in the vicinity of the leeward face decrease. This is because of the strong updrafts due to buoyancy (also seen in Fig. 4), especially close to the leeward face. Secondly, the strong updraft at lower Ri decreases the heat transport in the streamwise direction. It can be seen in the figure that the stream- 


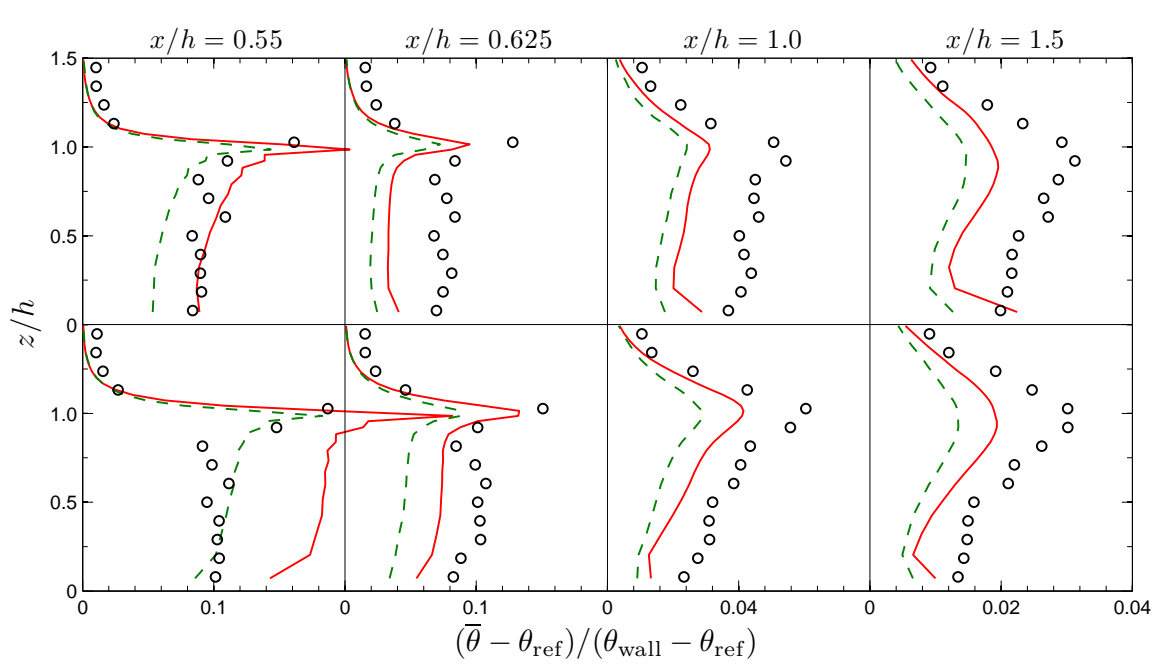

Fig. 5 Normalized mean temperature profiles for $R i=-0.66$ at different downstream locations behind the cube; solid lines - LES (with wall models given in Sect. 2.3), dashed lines - LES (with standard wall models), symbols - experiments; top row at $y / h=0$, bottom row - average of data at $y / h= \pm 0.4$.

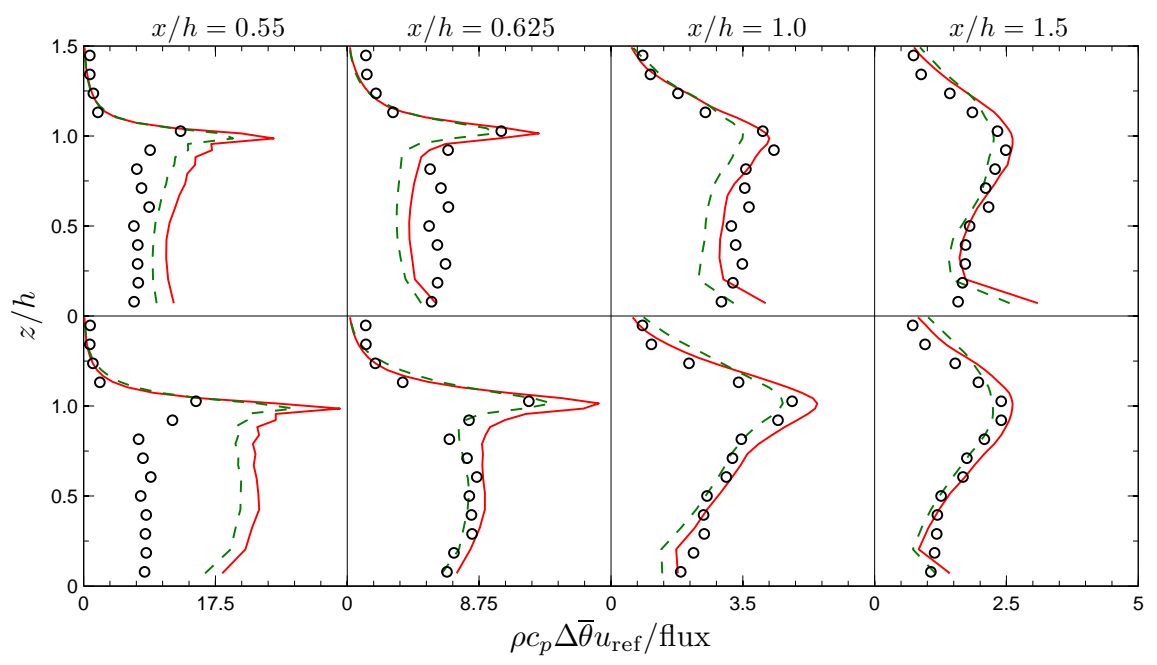

Fig. 6 Flux-normalized mean temperature profiles for $R i=-0.66$ at different downstream locations behind the cube; $\Delta \theta=\left(\bar{\theta}-\theta_{\text {ref }}\right)$; solid lines - LES (flux $\left.=q\right)$, dashed lines - LES (flux $=q_{\mathrm{est}}$ ), symbols experiments (flux $=q_{\text {est }}$ ); top row at $y / h=0$, bottom row - average of data at $y / h= \pm 0.4$.

wise convection of heat is much stronger at $R i=-0.02$, thereby suggesting that, for weakly unstable flows, heat is predominantly convected downstream.

The above comparisons with experiments suggest that our LES of the flow and thermal fields are reasonably good. Further analysis of the heat transport processes was performed to better understand the variations with different $R i$. This was all based 


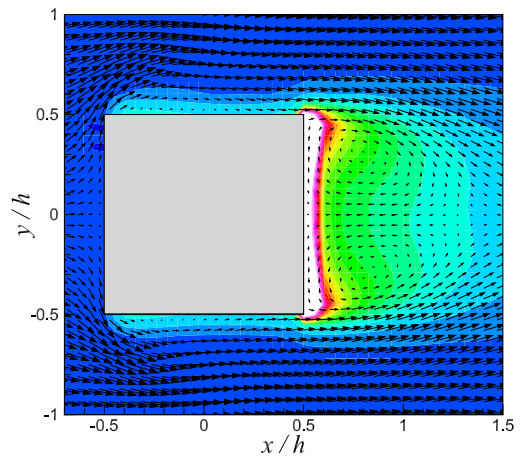

(a) $R i=-0.02$

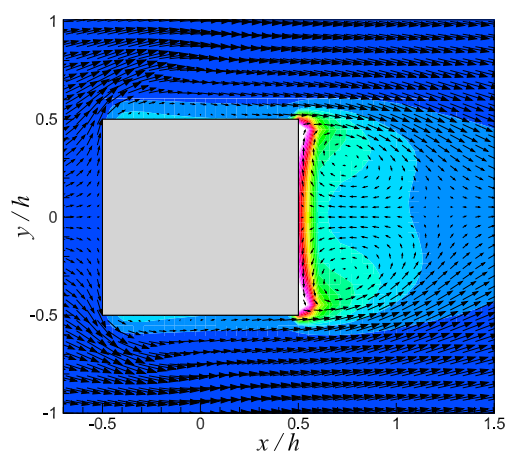

(c) $R i=-0.66$

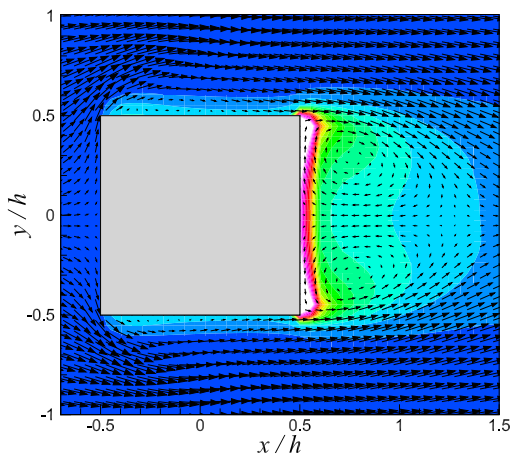

(b) $R i=-0.24$

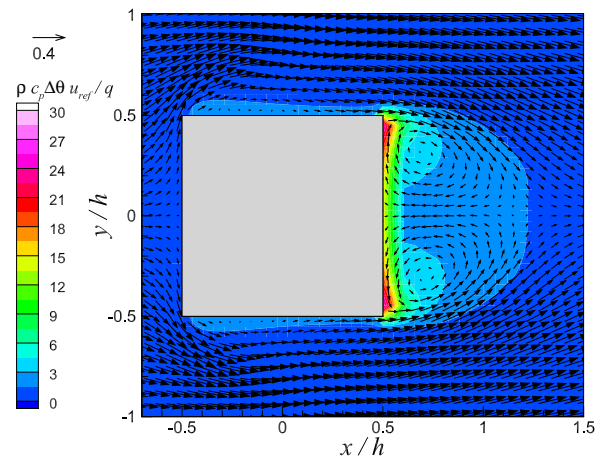

(d) $R i=-2$

Fig. 7 Contours of the flux-normalized mean temperature and normalized mean velocity vectors at $z / h=$ 0.5 .

on data from the computations with fixed temperature boundary conditions and is presented and discussed below.

301

302

3.2 Further analysis

\subsubsection{Fluxes}

In order to understand how the vertical distribution of heat fluxes vary with $R i$, each of its components i.e. advective, turbulent and diffusive fluxes are calculated. These 
are defined as:

$$
\begin{aligned}
\mathrm{A}_{\mathrm{f}} \equiv \text { advective heat flux }= & \rho c_{p}\left\{\frac{1}{L_{x} L_{y}} \int_{0}^{x} \int_{0}^{y} \tilde{\theta} \tilde{w} \mathrm{~d} x \mathrm{~d} y+\right. \\
& \left.\left(\frac{1}{L_{x} L_{y}} \int_{0}^{x} \int_{0}^{y} \bar{\theta} \mathrm{d} x \mathrm{~d} y\right)\left(\frac{1}{L_{x} L_{y}} \int_{0}^{x} \int_{0}^{y} \bar{w} \mathrm{~d} x \mathrm{~d} y\right)\right\}, \\
\mathrm{T}_{\mathrm{f}} \equiv \text { turbulent heat flux }= & \frac{\rho c_{p}}{L_{x} L_{y}} \int_{0}^{x} \int_{0}^{y} \overline{\theta^{\prime} w^{\prime}} \mathrm{d} x \mathrm{~d} y, \\
\mathrm{D}_{\mathrm{f}} \equiv \text { diffusive heat flux }= & \frac{\rho c_{p}}{L_{x} L_{y}} \int_{0}^{x} \int_{0}^{y}\left(k_{s}+k_{m}\right) \frac{\partial \bar{\theta}}{\partial z} \mathrm{~d} x \mathrm{~d} y .
\end{aligned}
$$

Note that $A_{f}$ and $T_{f}$ are resolved quantities, and the $k_{s}$ term in $D_{f}$ is the subgrid-scale modelled contribution of the total turbulent heat flux. The tilde variables are the deviations from the spatial mean of the time-average values, primed variables are the fluctuations from the respective time-averaged values and overbar denotes the time average. $L_{x}$ and $L_{y}$ are the respective domain lengths in the streamwise and spanwise directions. (Note, however, that to avoid the influence of the zero-gradient outlet boundary condition, we used an integration domain a little shorter than $L_{x}$.) The first term in the advective flux is called the dispersive flux and the contribution from the second term is found to be very small and hence is not shown in Fig. 8. The dispersive, turbulent and diffusive heat flux components shown in Fig. 8 are normalized by the total downwind convective heat flux obtained far downstream of the cube. It is observed that decreasing $R i$ leads to an increase in vertical dispersive flux and its maximum value gradually shifts towards the cube height. Due to the strong buoyancy effects at $R i=-0.66$ and -2.0 , the dispersive flux at the cube height is positive, in contrast to the negative values at $R i=-0.02$ and -0.24 . Below the cube height, the vertical turbulent flux values are very small and differ only marginally for all $R i$, suggesting that the fluctuations of the vertical velocity and temperature difference are poorly correlated. It can be observed that the maximum value of the turbulent heat flux decreases gradually and slightly shifts above the cube height with decrease in $R i$, again due to the stronger buoyancy effects at lower $R i$. Due to a reasonably good mesh resolution, the diffusive heat flux contribution for all $R i$ is found to be small compared to the other fluxes.

\subsubsection{Thermal boundary-layer thickness}

In the field study of Louka et al. (2002), it was observed that a large horizontal thermal gradient (at the vertical wall of the building) extends up to approximately $0.2 \mathrm{~m}$ from the wall and has a greater intensity at $0.02 \mathrm{~m}$ from the wall; the mean building height was $21 \mathrm{~m}$. Their measurements thus suggest that the thermal boundary layer is relatively very thin. However, it would be quite challenging to determine its precise thickness both in the field and in wind-tunnel models. We therefore estimate the 


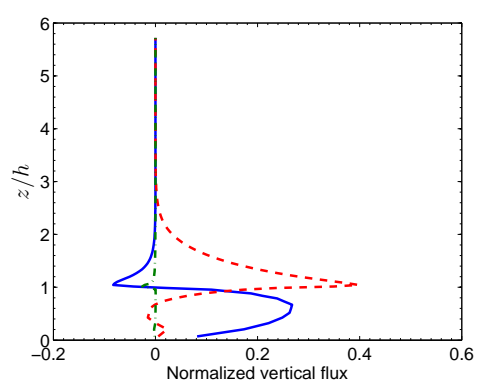

(a) $R i=-0.02$

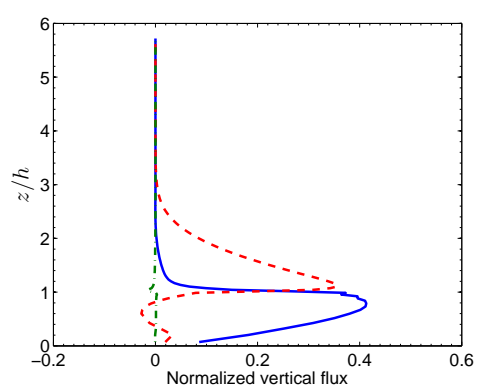

(c) $R i=-0.66$

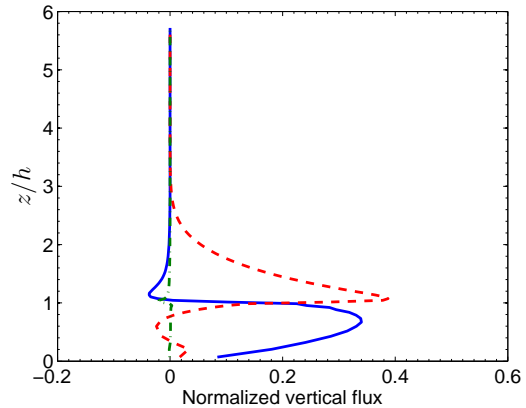

(b) $R i=-0.24$

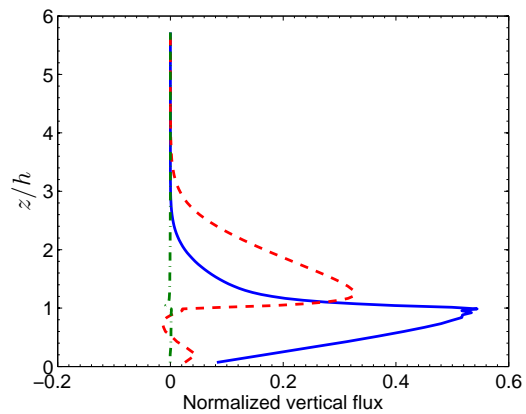

(d) $R i=-2$

Fig. 8 Normalized vertical heat flux profiles for different $R i$. Solid line: dispersive flux component of $A_{f}$, Dashed line: $T_{f}$, Dash-dot line: $D_{f}$.
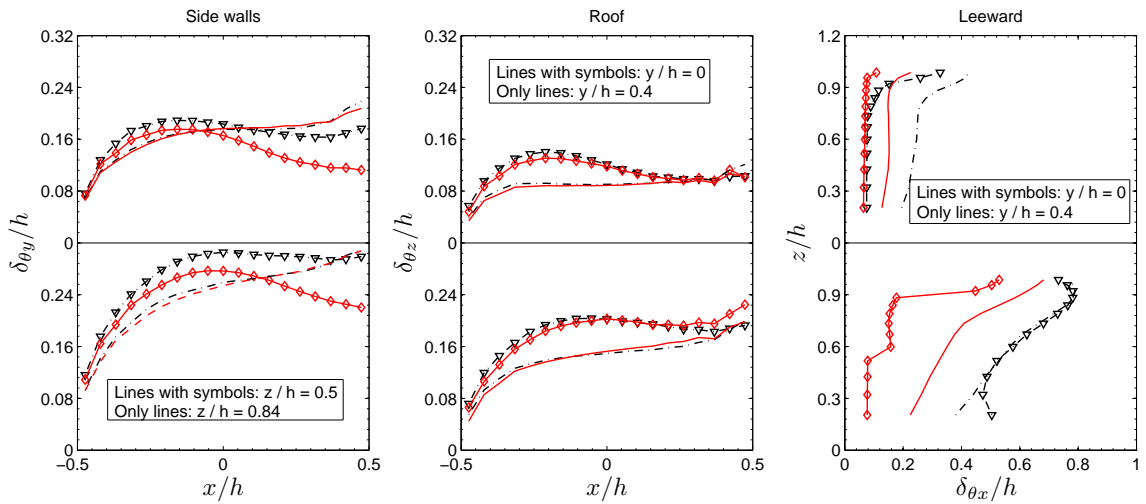

Fig. 9 Variation of thermal boundary-layer thickness at different locations. $R i=-0.24$ : dash-dot lines with and without triangles, $R i=-2$ : solid lines with and without diamonds. $\vartheta=0.05$ and 0.025 for top and bottom rows respectively. 
thermal boundary-layer thickness $\left(\delta_{\theta x_{i}}\right)$ in this section for various $R i$ and on different surfaces of the cube, where $\delta_{\theta x_{i}}$ is defined as the distance from the wall where $\left(\bar{\theta}-\theta_{\text {ref }}\right) /\left(\theta_{\text {wall }}-\theta_{\text {ref }}\right)=\vartheta$. The thickness therefore depends on the threshold value, $\vartheta$, and for comparison purposes, the threshold values chosen are 0.05 and 0.025. With this definition, $\delta_{\theta x_{i}}$ was estimated at different locations and for different $R i$. For brevity purpose, only the data that corresponds to $R i=-0.24$ and -2.0 are shown in Fig. 9. At $z / h=0.5$ and 0.84 the 'side' wall data correspond to the average values from two sides of the cube. Similarly the data at $y / h=0.4$ correspond to the average of values at $y / h=0.4$ and -0.4 . Details of the thermal thickness variations on each surface are explained below.

1. Side walls: The $\delta_{\theta y}$ variation along the side wall is shown at two different heights. At the cube's mid-height, it can be observed that $\delta_{\theta y} / h$ decreases with decrease in $R i$. This is due to the increase in buoyancy at lower $R i$. The larger thermal boundary-layer thickness at $R i=-0.24$ also indicates that the lateral dispersion of temperature is larger. This is also seen in Fig. 7. Towards the top of the cube i.e at $z / h=0.84, \delta_{\theta y}$ is almost the same for all $R i$ due to stronger streamwise velocity at that height. The presence of the recirculation region on the side walls, whose size decreases with decreasing $R i$, might be responsible for the slight bulging behaviour of $\delta_{\theta y}$ in the range $-0.5 \leq x / h \leq 0$ at $z / h=0.5$. Such bulging behaviour is very clear with $\vartheta=0.025$. It is to be noted that by decreasing $\vartheta$ from 0.05 to 0.025 , the thermal boundary-layer thickness increased approximately by $0.1 \mathrm{~h}$. This indicates the sensitivity of the thermal thickness to the threshold value.

2. Roof: The thermal thickness on the roof is smaller than on the sidewalls due to a larger streamwise velocity above the boundary layer on the roof. Because of the presence of a thin recirculation region which extends up to $x / h=0$ from the leading edge and whose length is almost same for all $R i$, the thermal thickness is larger at $y / h=0$ than it is at $y / h=0.4$. The sensitivity of the thermal thickness to $\vartheta$ is found to be similar to that of side walls.

3. Leeward: The thermal thickness for $z / h<0.2$ is not shown here as it is influenced by the floor heating. Due to stronger vertical convection at lower $R i$, a clear trend of decreasing thermal boundary-layer thickness with decreasing $R i$ can be observed. For $\vartheta=0.05$ and $0.025, \delta_{\theta x} / h$ is larger at $y / h=0.4$ than it is at the symmetry plane. The downstream convection of heat at $y / h=0$ is affected by the two counter rotating vortices on either side of the symmetry plane. Therefore $\delta_{\theta x} / h$ is smaller at $y / h=0$ than at $y / h=0.4$ and this is also very evident in Fig. 7. With decrease in $\vartheta$ to $0.025, \delta_{\theta x} / h$ increased by almost a factor of two, indicating the sensitivity of the thickness to the threshold value. It also suggests the presence of steeper horizontal thermal gradients, as observed in the field studies of Louka et al. (2002). Because of the wake flow behind the cube, the thermal boundary-layer thickness is greater on the rear wall than on the sidewalls and roof surfaces.

4. Windward: In order to estimate the thermal boundary-layer thickness on the windward face, an additional computation that includes some heating on the windward face was done for $R i=-0.66$. This implies that the whole cube is heated, but with different temperatures on each face (the sidewalls and windward face had 


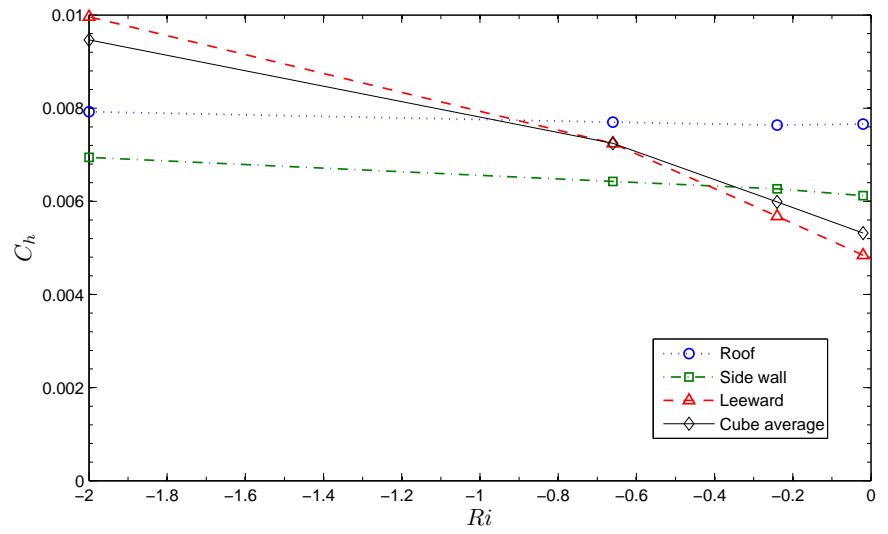

Fig. 10 Heat transfer coefficient at cube surfaces.

\begin{abstract}
the same temperature in this case). The thermal boundary layer is found to be thinner than $0.1 \mathrm{~h}$ for $\vartheta=0.025$ and not measurable (i.e. thinner than the distance to the first grid point away from the wall) for $\vartheta=0.05$. These relatively very thin boundary layers (compared to those on the other surfaces) are almost certainly caused by flow impingement on the windward surface.
\end{abstract}

\title{
3.2.3 Heat transfer coefficient
}

The convective heat transfer coefficient $\left(C_{h}\right)$ is an important parameter in urban microscale modelling. It's applications include assessment of energy performance of buildings, estimation of convective moisture transfer from building surfaces etc. Therefore, it would be useful to know the variation of $C_{h}$ for different heated surfaces of the cube and also for different $R i$. Here, it is defined as

$$
C_{h}=\frac{q_{\text {wall }}}{\rho c_{p} u_{b}\left(\theta_{\text {wall }}-\theta_{\text {ref }}\right)} .
$$

where $q_{\text {wall }}$ is the heat flux from each heated surface and $u_{b}$ is the bulk velocity of the mean flow. Recall that $\theta_{\text {wall }}$ varied on each heated surface (Table 1). We observe in Fig. 10 that, except for the leeward wall at $R i=-2$, the heat transfer coefficient at the roof surface is larger than sidewalls and leeward wall; this must be a result of strong streamwise convection. For the same reason, the values of $C_{h}$ are almost constant for all $R i$ on the roof surface. A marginal increase in $C_{h}$ can be seen at $R i=-2$ on side walls. On the leeward wall, $C_{h}$ is smaller than the roof and sidewalls for $R i=-0.02$ and -0.24 . But for $R i=-0.66, C_{h}$ for leeward wall is larger than on the sidewalls but smaller than on the roof. This suggests that the buoyancy effects are smaller than the streamwise convection on the roof surface, but larger than the sidewalls. For $R i=-2$, in contrast, $C_{h}$ on the leeward wall is larger than on the roof, indicating that the buoyancy effects are now dominant than the streamwise convection on the roof. 
The weighted average of the heat transfer coefficient at the heated surfaces of the cube is also shown in the same figure for each $R i$. The average heat transfer coefficient for $R i=-0.02$ is found to be approximately half of that observed in the wind-tunnel experiments by Meinders and Hanjalić (1999). In the COSMO (Comprehensive Outdoor Scale MOdel) field experiments by Kanda and Moriizumi (2009), the value of $C_{h}$ is estimated to be between 0.01 and 0.02 which is approximately 2 to 4 times larger than the current computations. These differences could be a result of all or some of the following: (i) both these experimental studies consisted of flow past an array of aligned cubes whereas the current LES computes flow past a single cube; (ii) the domain height in our LES is nearly three times larger than in the Meinders and Hanjalić (1999) (channel) experiments, which implies a higher bulk velocity; (iii) to obtain the values of $C_{h}$ in the COSMO field experiments, surface radiative temperature was used (at $z / h=3$ ), which is different from the local surface temperature. It could also be possible that $R i$ was very large in the COSMO experiments.

\subsubsection{Quadrant analysis}

A simple statistical tool that measures the contribution of different kinds of events arising from the large-scale coherent structures is 'quadrant analysis'. This technique has been used extensively in vegetation canopies and a detailed review by Finnigan (2000) shows that sweeps contribute more to the momentum flux as well as the heat flux within and just above the canopy. In the case of urban roughness, studies (e.g Rotach, 1993; Castro et al., 2006) also showed that momentum transfer close to and within the canopy is dominated by sweep events. Quadrant analysis by Christen et al. (2007) showed that the turbulent momentum and heat fluxes are predominantly by sweeps in most regions of the urban street canyon and closer to roofs whereas ejections were found to be slightly dominating at higher measurement locations. In contrast, Shiau and Hsieh (2002) found that for a shallow (low-slope) trapezium-shaped body in a wind tunnel, ejections were stronger than sweeps at $z / h=1.2$.

To find which kind of events dominate in the current simulations where the leeward wall of the cube was strongly heated, quadrant analysis was carried out on both momentum and heat transport. The natural classification of quadrant events is used and is given below.

$$
\begin{array}{ccc}
\text { Ejection }(\mathrm{E}): & u^{\prime}<0, w^{\prime}>0 ; & \theta^{\prime}>0, w^{\prime}>0 \\
\text { Sweep }(\mathrm{S}): & u^{\prime}>0, w^{\prime}<0 ; & \theta^{\prime}<0, w^{\prime}<0 \\
\text { Outward interaction }(\mathrm{O}): & u^{\prime}>0, w^{\prime}>0 ; & \theta^{\prime}<0, w^{\prime}>0 \\
\text { Inward interaction }(\mathrm{I}): & u^{\prime}<0, w^{\prime}<0 ; & \theta^{\prime}>0, w^{\prime}<0
\end{array}
$$

The primed quantities (i.e. $u^{\prime}, w^{\prime}, \theta^{\prime}$ ) denote fluctuations from the respective timemean values. Analogous to the momentum flux, the removal of hot air from the cube wake to the flow above is called an 'ejection (E)' and the in-rush of cold air upstream of the cube to its wake is called a 'sweep (S)'. The momentum and heat fluxes corre- 

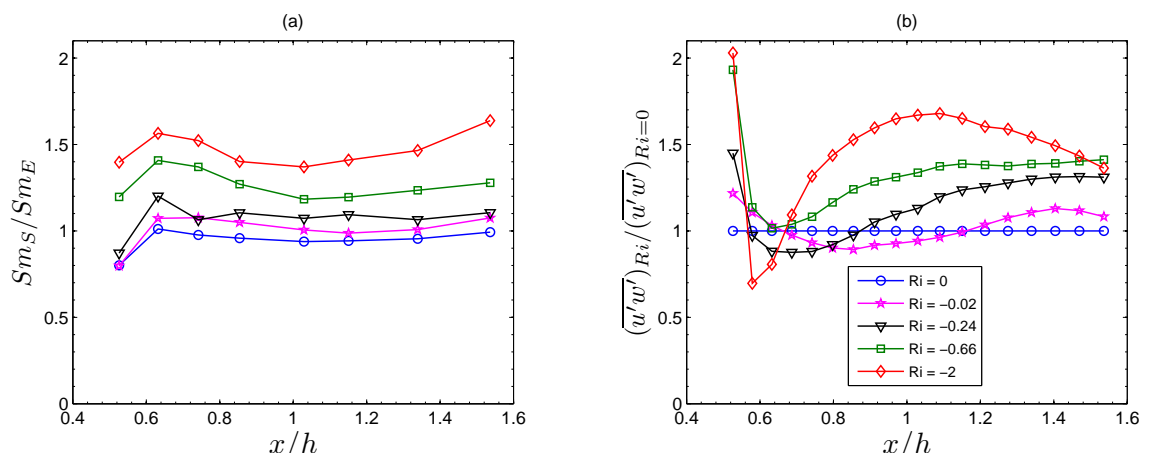

Fig. 11 Momentum flux along the shear layer behind the cube. (a) Ratio of strength of sweeps to ejections; (b) Variation of momentum flux with respect to neutral case. Legend in (b) refers to (a) also.

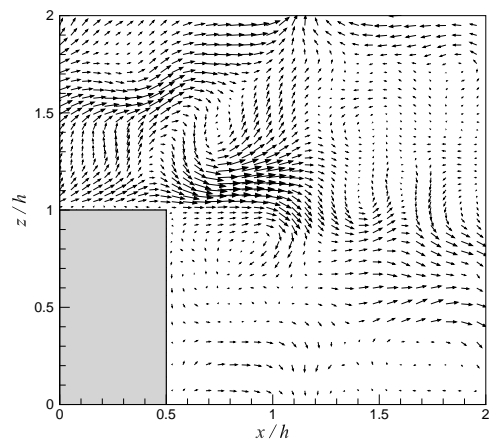

(a) $R i=0$

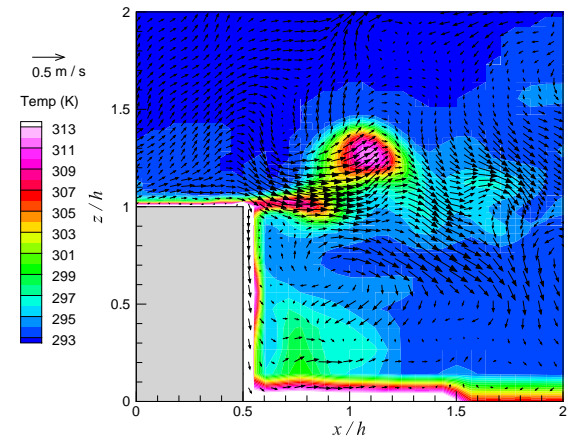

(b) $R i=-2$

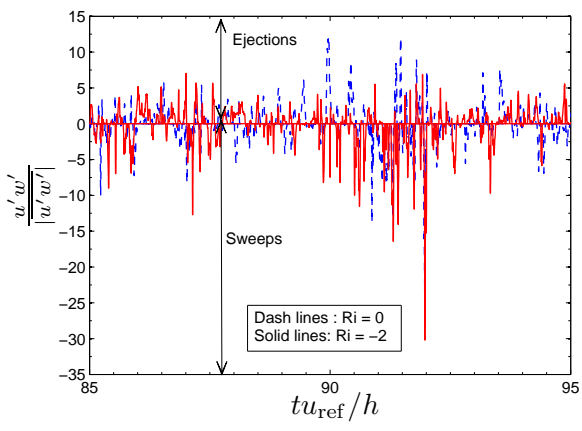

(c)

Fig. 12 The vector field of instantaneous velocity fluctuations (a \& b) and contour of the instantaneous temperature (b) and in the wake of the cube at $y / h=0$. (c) Time series of normalized shear-stress corresponding to the ejection and sweep events at $(x / h, z / h)=(1.54,1.01)$. For clarity the sign of shear stress for ejections is reversed. 
sponding to different quadrant events are given by

$$
\begin{aligned}
& \left(\overline{u^{\prime} w^{\prime}}\right)_{Q}=\frac{1}{T} \int_{T} u^{\prime}(t) w^{\prime}(t) I_{Q}(t) \mathrm{d} t \\
& \left(\overline{\theta^{\prime} w^{\prime}}\right)_{Q}=\frac{1}{T} \int_{T} \theta^{\prime}(t) w^{\prime}(t) I_{Q}(t) \mathrm{d} t
\end{aligned}
$$

where $Q$ denotes the quadrant event i.e. 'S', 'W', 'O' and 'I'. $I_{Q}(t)$ is an indicator function and is given by

$$
I_{Q}(t)=\left\{\begin{array}{c}
1 \text { if }\left(u^{\prime}, w^{\prime}\right) \text { is in quadrant } Q \\
\text { if }\left(\theta^{\prime}, w^{\prime}\right) \text { is in quadrant } Q \\
0 \text { otherwise }
\end{array}\right.
$$

The event frequency and the stress and heat flux fractions contributed by the individual events are then given by

$$
\begin{aligned}
F_{Q} & =\frac{1}{T} \int_{T} I_{Q}(t) \mathrm{d} t, \\
S m_{Q} & =\frac{\left(\overline{u^{\prime} w^{\prime}}\right)_{Q}}{\overline{u^{\prime} w^{\prime}}}, \\
S h_{Q} & =\frac{\left(\overline{\theta^{\prime} w^{\prime}}\right)_{Q}}{\overline{\theta^{\prime} w^{\prime}}}
\end{aligned}
$$

Momentum flux contributions from the four quadrant events are shown along the shear layer and at the symmetry plane i.e. $(y / h, z / h)=(0,1)$ in Fig. 11a. It is observed that sweep ('S') and ejection ('E') events occur more frequently than outward ('O') or inward ('I') interaction events in the case of momentum transport. With decrease in $R i$, a gradual increase in the frequency of ' $\mathrm{O}$ ' and ' $\mathrm{I}$ ' events is observed, but these are still dominated by the frequency of ' $S$ ' and ' $E$ ' events. Although ' $E$ ' events occur more frequently than ' $S$ ' events, the latter contributes more to the momentum flux. Also, this contribution increases with decrease in $R i$ and this can be seen in Fig. 11a. This could be due to the displacement of cold, denser air flowing from upstream by the thermal plume emanating from the leeward face. The former is then subjected to stronger fluctuations in the downward direction thereby yielding stronger sweeps. This can be seen in Fig. 12b and for comparison purposes the neutral case is shown in Fig. 12a. Although, this figure pertains to one specific time instant, a snapshot of the time series for $R i=0$ and -2 , shown in Fig. 12c, confirms that heating enhances the sweep strength considerably in the shear layer. To clarify the behaviour of the momentum flux along the shear layer with decrease in $R i$, it is normalized with respect to the neutral case data and this is shown in Fig. 11b. It is evident that heating increases the normalized momentum flux, but in a non-monotonic way.

Unlike momentum, the frequency of the events that correspond to ' $S$ ' and ' $E$ ' in the heat flux transport dominate ' $\mathrm{O}$ ' and ' $\mathrm{I}$ ' events only for $R i=-0.02$ and -0.24 . For lower $R i$ this behaviour changes and, as an example, the frequency distribution of the events, $F_{Q}$, is shown for $R i=-2$ in Fig. $13 \mathrm{c}$. In comparison with $F_{Q}$ for momentum in 

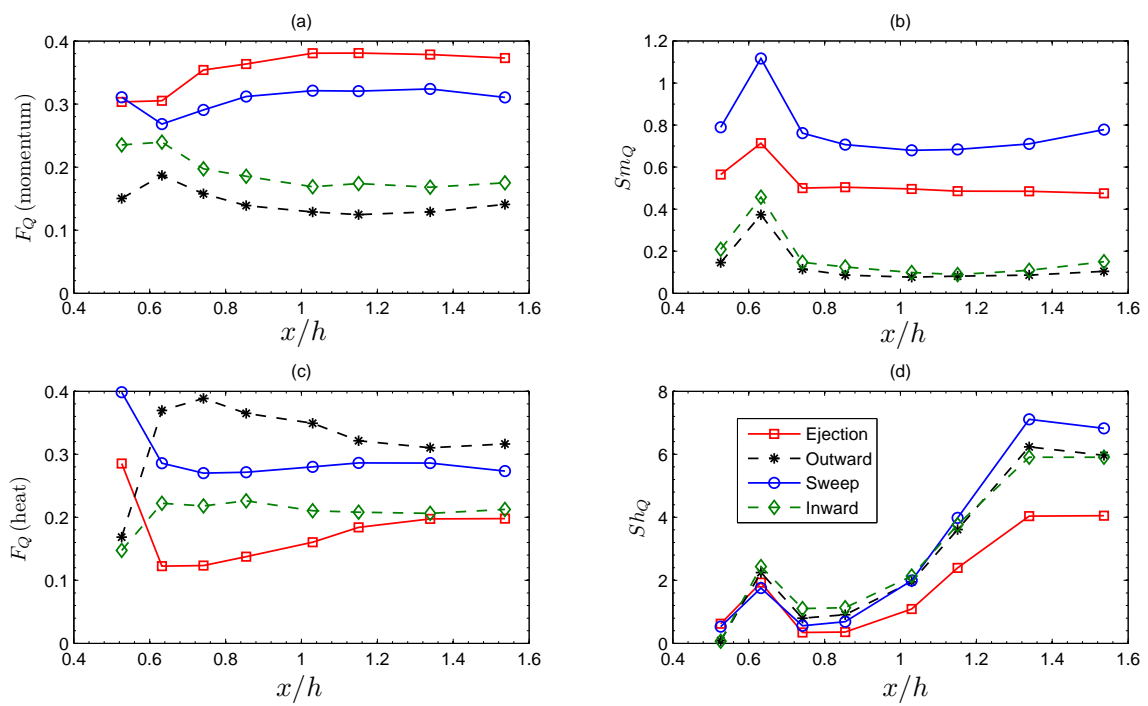

Fig. 13 The frequency $(a, c)$ and flux fractions $(b, d)$ of momentum and heat along the shear layer for $R i=-2$. Note that the signs of stress fractions of outward and inward interactions for momentum and heat in (b) and (d) are reversed for better comparison with the ejection and sweep events.

Fig. 13a where the frequencies of ' $\mathrm{E}$ ' and ' $\mathrm{O}$ ' are the highest and lowest respectively, the $F_{Q}$ of heat in Fig. 13c shows the opposite behaviour. As expected, the heat flux fraction of the ' $E$ ' events $\left(S h_{E}\right)$ is higher in the vicinity of the leeward wall. The larger values of $S h_{S}$ for $x / h>1$ in Fig. 13d could indicate a strong in-rush of cold air into the shear layer. Unlike the momentum flux distribution in Fig. 13b, where the ' $\mathrm{O}$ ' and 'I' events are weaker than the ' $E$ ' and ' $S$ ' events all along the shear layer, Fig. 13d shows that the strengths of ' $O$ ' and ' $I$ ' lie between those of ' $E$ ' and ' $S$ ' events for $x / h>1$; for $x / h<1$ the strengths of ' $\mathrm{O}$ ' and 'I' events are found to be larger. All this suggests that the contributions to net heat flux from the different quadrants are very different to those of shear stress.

The normalized vertical turbulent heat flux variations along the shear layer for various $R i$ are shown in Fig. 14. We observe that with increase in heating, the normalized turbulent heat flux decreases and reaches a constant value as the distance from the leeward face increases. This decrease is caused by an increase in the heat flux contribution from the ' $\mathrm{O}$ ' and 'I' quadrant events with decrease in $R i$.

The above observations are valid within the very thin shear layer at the cube height. Corresponding quadrant analysis was also done at $(x, y, z) \approx\left(0.75 x_{R}, 0, z_{\mathrm{TKE}_{\mathrm{m}}}\right)$, where $x_{R}$ is the recirculation length and $z_{\mathrm{TKE}_{\mathrm{m}}}$ is the height at which turbulent kinetic energy is maximum; both these values vary with $R i$. This analysis showed that the strength of ejections dominates that of sweeps, but only marginally for momentum transport. This is in contrast to the above observation made for the shear layer region. However, in the case of heat flux, sweeps continued to dominate ejections, with increasing strength for decreasing $R i$. The above quadrant analysis gives a general overview of the kind of events that arise in thin shear layer region behind the cube, 


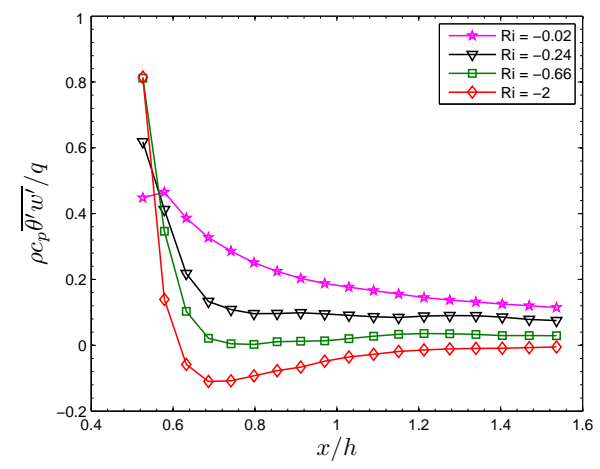

Fig. 14 Variation of normalized turbulent heat flux along the shear layer.

with strong leeward wall heating. The findings are found to be similar to those observed in field and wind-tunnel studies mentioned earlier, particularly for momentum.

\section{Conclusions}

The heat transfer from a single building with strong heating on its leeward surface and surrounded by an urban roughness array has been studied using LES for various degrees of thermal forcing ranging from neutral to weakly unstable to highly unstable flows around the building. The (neutrally stable) turbulent atmospheric boundary layer consists of a constant shear-stress region up to half of the boundary-layer depth. It is quite challenging, both in the wind tunnel as well as in numerical simulations, to generate such a deep surface layer. But with the inflow boundary condition developed by Xie and Castro (2008), it is possible to generate such an approach flow in the LES. Three sets of wall models are used: (i) a rough-wall model to represent an aligned array of roughness elements on the floor of the wind tunnel; (ii) a momentum wall model, and (iii) a temperature wall model to improve the flow and temperature estimation near the smooth wall surfaces on a relatively coarse mesh. The resulting flow and thermal fields showed fair agreement with the wind-tunnel experiments. The buoyancy effects are found to be significant in the wake of the cube, especially at lower $R i$. At higher $R i$, the heat emitted from the cube is found to be concentrated at the leeward wall and convected downwind.

The thermal boundary-layer thickness and heat transfer coefficient are estimated for each heated surface of the cube and for all $R i$. It is observed that the thermal boundary layers on the side and leeward walls become thinner with a decrease in $R i$. However, compared to the side walls and roof surface, the thermal boundary layer on the leeward wall is found to be larger because of the weak streamwise convection. Irrespective of the variation in $R i$, the stronger streamwise convection on the top of the cube yielded a thinner boundary layer compared to the other surfaces. For the same reason, the heat transfer coefficient is found to be largest on the roof surface, except for $R i=-2$ where the buoyancy effects resulted in the largest heat transfer coefficient at the leeward wall. This suggests that, at such low $R i$, buoyancy dominates the effects 
of the strong shear layer normally present at roof level. These details should be useful in the development or improvement of empirical models of heat transfer around buildings in urban areas. The quadrant analysis performed along the shear layer behind the cube showed that the momentum flux is mostly due to stronger sweeps and that heating increases the sweep strength considerably (relative to ejections). In the case of heat, the quadrant analysis showed a similar behaviour to momentum only for relatively high $R i=-0.02$ and -0.24 . Unlike the momentum flux, the frequency and strength of the heat flux from the outward and inward interaction quadrants are not small. This suggests that at lower $R i$ the contribution of these events to the heat flux is very different to that for the momentum flux.

Whilst this investigation has focussed on the flow and thermal fields in the wake of a single heated building, partly because of the availability of a corresponding experimental dataset, extension to arrays of urban-like roughness elements with heating analogous to, for example, the incidence of solar radiation will clearly be important. This will be the subject of future work, in which we hope to explore the integrated effects of surface heating on momentum transfer and turbulence in urban-like areas.

Acknowledgements This project is funded by the Natural Environment Research Council, through its National Centre for Atmospheric Sciences, Grant No. R8/H12/38. We thank Prof. Dr. Bernd Leitl of Universität Hamburg for providing the experimental data. We thank the referees for their valuable comments. All computations have been done using CD-adapco's STAR-CD code on Iridis (University's supercomputer) and HECToR (U.K's supercomputer). We thank the CD-adapco's staff Fred Mendonca for his continuous support and Stefano Duranti for helping us to implement the wall models in Star-CD

\section{A Appendix: Modelling the approach flow in a channel}

In the experiments, the model scale of the cube was 1:100. A turbulent atmospheric boundary layer was simulated such that the turbulent energy and shear stress were both essentially constant to a height equal to one half of the boundary-layer height. In the computations, it is crucial to maintain the approach flow used in the experiments but it is equally challenging to create and maintain a developed boundary-layer profile with the necessary constant turbulent energy and shear stress in a deep surface layer over an axial fetch significantly larger than the cube dimension. Initial computations were performed with a bottom rough wall and without the cube, to check that the required flow did not change significantly with an upwind fetch.

The computational domain size of the channel flow was $18 h \times 9 h \times 6 h$. A height of $6 h$ was deemed sufficient, since that is similar to the boundary-layer thickness in the experiments. The inlet velocity profile in the experiments followed a power-law and the same was specified in the computations. Reynolds normal stress components were specified at the inlet such that the turbulent kinetic energy (TKE) agreed with that of the experiments. The Reynolds shear stress specified at the inlet had a constant value in the surface layer with a decreasing linear profile above. Cyclic boundary conditions were employed in the spanwise direction. The bottom rough wall was represented by the rough-wall model described in Sect. 2.3.1. Stress free conditions were specified on the top surface and, at the outlet, the axial gradient of the velocity field was set to zero followed by a mass balance correction. The resulting profiles of time-averaged normalized streamwise velocity and TKE are shown in Fig. 15. It can be seen that the streamwise velocity increases only marginally with increasing downstream distance. However, the turbulent kinetic energy profiles at various downstream locations indicate that the flow is developing, although very slowly (especially when compared to RANS computations not shown here). The sharp peaks in the kinetic energy at the second wall grid point in Fig. 15b could be due to the injection of energy into the resolved velocity field at the 

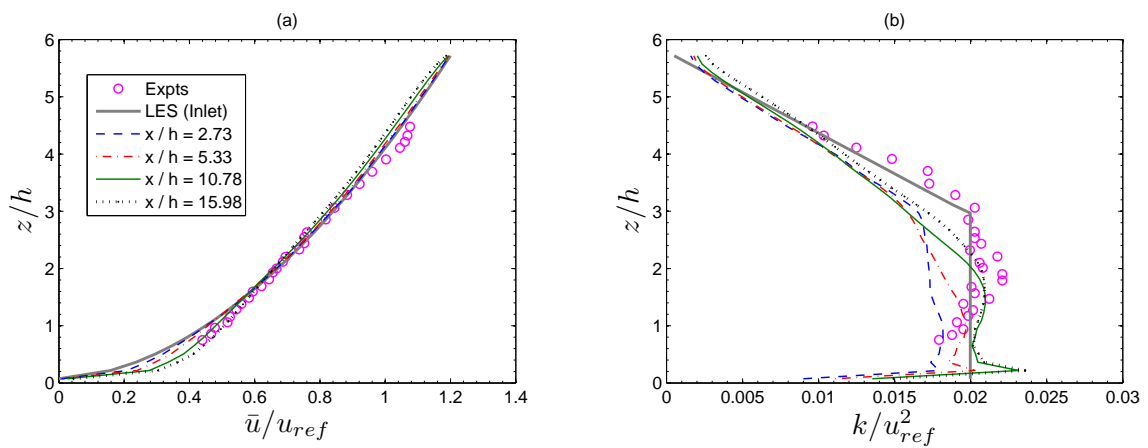

Fig. 15 (a) Normalized mean streamwise velocity and (b) turbulent kinetic energy profiles at various downstream locations.

wall surface by the rough-wall model. Despite these small imperfections, it is clear that an approach flow similar to the experiments can be adequately represented with the inflow and rough-wall models.

\section{References}

Boppana VBL, Xie ZT, Castro IP (2010) Large-eddy simulation of dispersion from surface sources in arrays of obstacles. Boundary-Layer Meteorol 135:433-454

Cabot W, Moin P (1999) Approximate wall boundary conditions in the large-eddy simulation of high reynolds number flow. Flow, Turbul Combust 63:269-291

Cai X (2012) Effects of differential wall heating in street canyons on dispersion and ventilation characteristics of a passive scalar. Atmos Environ 51:268-277

Castro IP, Cheng H, Reynolds R (2006) Turbulence over urban-type roughness: deductions from wind-tunnel measurements. Boundary-Layer Meteorol 118:109_ 131

Cheng WC, Liu CH (2011) Large-eddy simulation of turbulent transports in urban street canyons in different thermal stabilities. J Wind Eng Ind Aerodyn 99:434 442

Christen A, van Gorsel E, Vogt R (2007) Coherent structures in urban roughness sublayer turbulence. Int J Climatol 27:1955-1968

Dimitrova R, Sini JF, Richards K, Schatzmann M, Weeks M, Garcia EP, Borrego C (2009) Influence of thermal effects on the wind field within the urban environment. Boundary-Layer Meteorol 131:223-243

Finnigan J (2000) Turbulence in plant canopies. Annu Rev Fluid Mech 32:519-571

Idczak M, Mestayer P, Rosant JM, Sini JF, Violleau M (2007) Micrometeorological measurements in a street canyon during the joing ATREUS-PICADA experiment. Boundary-Layer Meteorol 124:25-41

Kanda M, Moriizumi T (2009) Momentum and heat transfer over urban-like surfaces. Boundary-Layer Meteorol 131:385-401

Kim JJ, Baik JJ (1999) A numerical study of thermal effects on flow and pollutant dispersion in urban street canyons. J App Meteorol 38:1249-1261 
Li XX, Britter RE, Koh TY, Norford LK, Liu CH, Entekhabi A, Leung DYC (2010) Large-eddy simulation of flow and pollutant transport in urban street canyons with ground heating. Boundary-Layer Meteorol 137:187-204

Lim HC, Thoma TG, Castro IP (2009) Flow around a cube in a turbulent boundary layer: LES and experiment. J Wind Eng Ind Aerodyn 97:96-109

Louka P, Vachon G, Sini JF, Mestayer PG, Rosant JM (2002) Thermal effects on the airflow in a street canyon-Nantes' 99 experimental results and model simulations. Water Air Soil Pollut 2:351-364

Meinders ER, Hanjalić K (1999) Vortex structure and heat transfer in turbulent flow over a wall-mounted matrix of cubes. Int J Heat and Fluid Flow 20:255-267

Nottrott A, Onomura S, Inagaki A, Kanda M, Kleissl J (2011) Convective heat transfer on leeward building walls in an urban environment: Measurements in an outdoor scale model. Int J Heat and Mass Transfer 54:3128-3138

Offerle O, Eliasson I, Grimmond CSB, Holmer B (2007) Surface heating in relation to air temperature, wind and turbulence in an urban street canyon. Boundary-Layer Meteorol 122:273-292

Park SB, Baik JJ, Raasch S, Letzel MO (2012) A large-eddy simulation study of thermal effects on turbulent flow and dispersion in and above a street canyon. $\mathrm{J}$ Appl Meteorol and Climatol 51:829-841

Pascheke F, Barlow JF, Robins A (2008) Wind-tunnel modelling of dispersion from a scalar area source in urban-like roughness. Boundary-Layer Meteorol 126:103124

Rani SL, Smith CE, Nix AC (2009) Boundary-layer equation-based wall model for large-eddy simulation of turbulent flows with wall heat transfer. Num Heat Transfer, Part B 55:91-115

Richards K, Schatzmann M, Leitl B (2006) Wind tunnel experiments modelling the thermal effects within the vicinity of a single block building with leeward wall heating. J Wind Eng Ind Aerodyn 94:621-636

Rotach MW (1993) Turbulence close to a rough urban surface part I: Reynolds stress. Boundary-Layer Meteorol 65:1-28

Shiau BS, Hsieh CT (2002) Wind flow characteristics and reynolds stress structure around the two-dimensional embankment of trapezoidal shape with different slope gradients. J Wind Eng Ind Aerodyn 90:1645-1656

Sini JF, Anquetin S, Mestayer PG (1996) Pollutant dispersion and thermal effects in urban street canyons. Atmos Environ 30:2659-2677

Wang M, Moin P (2002) Dynamic wall modeling for large-eddy simulation of complex turbulent flows. Phys Fluids 14:2043-2051

Xie ZT, Castro IP (2008) Efficient generation of inflow conditions for large eddy simulation of street-scale flows. Flow Turb Combust 81:449-470

Xie ZT, Voke PR, Hayden P, Robins AG (2004) Large-eddy simulation of turbulent flow over a rough surface. Boundary-Layer Meteorol 111:417-440 